\title{
Role of microRNA-33a/b in Cholesterol Metabolism in Type 2 Diabetic Patients in Ouagadougou, Burkina Faso
}

\author{
Alice Kiba Koumaré ${ }^{1,2,3, \text { * }}$, Tegwinde Rebeca Compaoré ${ }^{3}$, Fabienne Soudré ${ }^{1}$, Raoul Karfo ${ }^{1}$, \\ Gnabôrou Rachid Konfé ${ }^{2}$, Elie Kabré ${ }^{1,2}$, Ignatius Baldeh ${ }^{5}$, Jacques Simporé ${ }^{3,4}$, Jean Sakandé ${ }^{1,2}$ \\ ${ }^{1}$ Biochemistry Department, Faculty of Health Sciences, University Joseph Ki-Zerbo, Ouagadougou, Burkina Faso \\ ${ }^{2}$ Laboratory Department, University Hospital Yalgado Ouedraogo, Ouagadougou, Burkina Faso \\ ${ }^{3}$ Molecular Biology Department, Centre of Biomolecular Research Pietro Annigoni (CEBRA), Ouagadougou, Burkina Faso \\ ${ }^{4}$ Biology and Genetics Department, University Joseph Ki-Zerbo, Ouagadougou, Burkina Faso \\ ${ }^{5}$ Clinical Laboratory Department, National Public Health Laborator Y, Banjul, Gambia
}

\section{Email address:}

alice_kiba@yahoo.fr (A. K. Koumaré), rebecca23fr@yahoo.fr (T. R. Compaoré), fabysoudre@gmail.com (F. Soudré), rkarf006@yahoo.fr (R. Karfo), konfegrachid@gmail.com (G. R. Konfé), elie.kabre@gmail.com (E. Kabré), baldehignatius@gmail.com (I. Baldeh),jacques.simpore@yahoo.fr (J. Simporé), jsakande@gmail.com (J. Sakandé) ${ }^{*}$ Corresponding author

\section{To cite this article:}

Alice Kiba Koumaré, Tegwinde Rebeca Compaoré, Fabienne Soudré, Raoul Karfo, Gnabôrou. Rachid Konfé, Elie Kabré, Ignatius Baldeh, Jacques Simporé, Jean Sakandé. Role of microRNA-33a/b in Cholesterol Metabolism in Type 2 Diabetic Patients in Ouagadougou, Burkina Faso. Advances in Biochemistry. Vol. 7, No. 4, 2019, pp. 71-76. doi: 10.11648/j.ab.20190704.11

Received: December 8, 2019; Accepted: December 27, 2019; Published: January 4, 2020

\begin{abstract}
MicroRNAs (miRNAs) are small endogenous RNAs approximately 22 nucleotides involved in the regulation of several cellular metabolisms including cholesterol metabolism. The objective of this study was to measure miRNAs 33a and $33 \mathrm{~b}$ in type 2 diabetics to evaluate their impact on the lipids levels and prevalence of dyslipidemia. The study population profile was 45 subjects including 30 type 2 diabetic patients and 15 healthy controls. The lipids tests were performed using an automated Spintech 240 Biolis analyzer and the microRNAs (33a and 33b) by applied biosystems 7500 Fast Real Time PCR System using the TaqMan ${ }^{\circledR}$ MicroRNA Assay kit. The prevalence of dyslipidemia was higher in miRNA-33a positive subjects than miRNA-33a negative $(\mathrm{p}<0.0001)$. The prevalence of dyslipidemia was however not significant between miRNA-33b positive and miRNA-33b negative. A comparison between miRNA-33a positive and miRNA-33b positive showed a significant increase of dyslipidemia in miRNA-33a positive than in miRNA-33b positive subjects. The dyslipidemic types in miRNA-33a positive diabetics were $90 \%$ hypercholesterolemia, $88 \%$ LDLC increase and $83.33 \%$ HDLC decrease. The measurement of the HDLC subclasses showed $82.6 \%$ HDL2C decrease and $90.91 \%$ HDL3C increase. The HDL3C level increased in $100 \%$ of non hypertensive diabetics versus $46.67 \%$ in hypertensive diabetics $(\mathrm{p}=0.003)$. The increase of HDL3C was $90.9 \%$ in miRNA33a positive subjects versus $54.5 \%$ in miRNA33b positive subjects $(\mathrm{p}<0.006)$. The study therefore confirms the relationship between the presence of microRNAs 33a and increased cardiovascular risk. The results showed a role of microRNA-33a on the increase of HDL3C which has a weak atheroprotective role compared to HDL2C. This observation suggests that the research on drugs able to increase the HDLC level based on microRNA regulation should target the stimulation of HDL2C synthesis.
\end{abstract}

Keywords: Type 2 Diabetes, miRNA-33a / b, Dyslipidemia, Ouagadougou

\section{Introduction}

MiroRNAs (miRNAs) are small endogenous RNAs approximately 22 nucleotides in length that have emerged as important post-transcriptional regulators of different protein- coding genes. It was first discovered in the nematode Caenoshabditis elegans, and since then has been identified in the genomes of most plants, animal, and viruses [1]. Some miRNAs have been identified to be potent posttranscriptional regulators of lipid metabolism genes, 
including miR-122, miR-33, miR-758, and miR-106b. The miR-33 over-expression strongly represses ABCA1 (the ATPbinding transporter 1) expression at the RNA and protein level and decreases cellular cholesterol efflux to apolipor protein A-I (ApoA-I), a key step in regulating reverse cholesterol transport (RCT). Conversely, antagonism of miR33 upregulates ABCA1 expression in vitro and in vivo and promotes cholesterol efflux to ApoA-I. Importantly, in vivo inhibition of miR-33 expression leads to a significant increase in plasma HDL levels and the regression of atherosclerosis, thus confirming the physiological effects of miR-33 in regulating lipid metabolism [2-4]. The mir-33a and mir-33b, intronic miRNAs located within the Serbp 2 and Srebpl genes, respectively [5] are co-transcribed with their host genes and regulate cholesterol and fatty acid metabolism. In previous studies in Burkina Faso [6-7], the measurement of cholesterol sub-fractions reported a significant increase in the total cholesterol (TC), the high density lipoprotein cholesterol (HDLC) and HDL3 cholesterol (HDL3C) in type 2 diabetes and in hypertensive patients compared to a control group. On the contrary, a significant decrease of HDL2 cholesterol (HDL2C) was observed in these same two groups. Particularly, the HDL2C decrease was significantly associated with diabetes complications. This observation was consistent with another study reporting that HDLC of hypertensive patients was markedly increased relative to their HDL3C fraction while their HDL2C fraction was reduced by $50 \%$ [8]. This study aimed to measure miRNA 33a and 33b in diabetics type 2 patients to evaluate their impact on lipids levels and the prevalence of dyslipidemia.

\section{Patients and Methods}

\subsection{Study Design and Subjects}

This was a case-control study conducted between October, 2017 to March, 2018 in Ouagadougou the capital city of Burkina Faso (West Africa). Type 2 Diabetes Mellitus patients were recruited at the University Hospital Yalgado Ouedraogo, Ouagadougou and the control group at the Regional Center of Blood Transfusion, Ouagadougou. Included in the study were newly and previously diagnosed type 2 diabetes patients with fasting blood glucose (FBG) $>7$ $\mathrm{mmol} / \mathrm{L}$. Subjects with or without complications of hypertension and attending the University Hospital Yalgado Ouedraogo were included. Apparently healthy nonhypertensive, non-diabetic controls were recruited at the Regional Center of Blood Transfusion of Ouagadougou. Among the type 2 diabetes patients, treatment with any lipid medication (statins, nicotinic acid, fibrate, resins) were excluded. Also, subjects should not have been enlisted in another concomitant study.

The study protocol and consent procedures were approved by the Burkina Faso National Ethics Committee for Research, Ouagadougou, Burkina Faso (approval No. 2017-09-78) on the 28th September, 2017. As required by the 1964 Helsinki declaration, a written informed consent was obtained from all participants prior to conducting any study procedure. After consenting, personal and epidemiological data were collected and recorded. All the data used in this study was anonymous.

\subsection{Lipids Analysis}

The lipids tests were performed at the University Hospital laboratory Yalgado Ouedraogo. After an overnight fast, venous blood was collected in a dry tube for biochemical analysis. Serum was separated by centrifugation at $3000 \mathrm{~g}$ for $10 \mathrm{~min}$ at $4^{\circ} \mathrm{C}$ and stored at $-80^{\circ} \mathrm{C}$. Testing was performed within a week. Serum levels for Total Cholesterol (TC) and Triglycerides (TG) were determined using an automated Spintech 240 Biolis 24j analyzer (Spintech, Barcelona, Span) and the fully enzymatic methods (Spinreact kits CholesterolLQ reference TK41021 and Triglycerides-LQ reference TK41031). The dual-step precipitation of HDL subfractions was performed according to the procedure described by Hirano et al. [9]. To isolate total HDLC by precipitation, a combined precipitant consisting of $100 \mu \mathrm{L}(0.02 \mathrm{mmol} / \mathrm{L})$ of dextran sulfate (Mr 500000, SIGMA, France) and $25 \mu \mathrm{L}$ (200mmol/L) of $\mathrm{MnCl} 2$ (MgCl2.6H2O, MERCK, France) was added to $1 \mathrm{~mL}$ of serum. After $15 \mathrm{~min}$ of standing at room temperature, the mixture was centrifuged at $3,400 \mathrm{~g}$ for $20 \mathrm{~min}$ at $4^{\circ} \mathrm{C}$. Aliquots of the resulting supernatant (S1) were collected for measuring HDLC levels and precipitation of HDL2C. The HDL2C was precipitated by a combined precipitant consisting of $100 \mu \mathrm{L}(0.02 \mathrm{mmol} / \mathrm{L})$ of dextran sulfate (Mr 500000, SIGMA, France) and 50 $\mu \mathrm{L}(200 \mathrm{mmol} / \mathrm{L})$ of $\mathrm{MnCl} 2(\mathrm{MgCl} 2.6 \mathrm{H} 2 \mathrm{O}, \mathrm{MERCK}$, France) added to $500 \mu \mathrm{L}$ of supernatant (S1). After $2 \mathrm{hrs}$ at room temperature, the mixture was centrifuged at $3,400 \mathrm{~g}$ for $20 \mathrm{~min}$ at $4^{\circ} \mathrm{C}$. Aliquots of the resulting supernatant (S2) were taken for measuring HDL3C levels. The measured value for total HDLC was multiplied by 1.125 and that for HDL3C was multiplied by 2.92 to correct for reagents dilutions. HDL3C was measured by the direct HDLC homogenous assay instead of the original TC assay. The sub-fraction HDL2C was calculated using the following formula: HDL2C $=$ HDLC-HDL3C. The LDL cholesterol (LDLC) was calculated in $\mathrm{mmol} / \mathrm{L}$ by using the Friedewald formula: LDLC $=$ TC-HDLC-TG/2.2 [10]. Dyslipidemia has been defined for $\mathrm{TC}>4.67 \mathrm{mmol} / \mathrm{L}$, $\mathrm{LDL}>2.55 \mathrm{mmol} / \mathrm{L}, \mathrm{HDL}<1.15 \mathrm{mmol} / \mathrm{L}, \mathrm{HDL} 2<0.43 \mathrm{mmol} / \mathrm{L}$, HDL $3>0.68 \mathrm{mmol} / \mathrm{L}$ according to the reference ranges of plasma lipids in Burkina Faso [11].

\section{3. miRNA Analysis}

The miRNA assay was performed at the Pietro Annigoni Biomolecular Research Center (CERBA), Ouagadougou. Total miRNA extraction was performed using the "mirVana TM miRNA" extraction kit (reference AM1560) according to the manufacturer's instructions. After total miRNA extraction, reverse transcription (RT) of the specific microRNAs (33a and 33b) using the "TaqMan ${ }^{\circledR}$ MicroRNA Assay kit" was immediately performed to obtain the complementary DNA (cDNA) from the total miRNA samples. Finally, a real-time 
PCR (qPCR) was performed from the cDNA samples using the applied biosystems 7500 Fast Real Time PCR System.

\subsection{Statistical Analysis}

Quantitative variables were expressed as Means \pm SD and qualitative variables in percentages. The Analysis of Variance (ANOVA) was used to determine quantitative variables with normal distribution, followed by the Bonferonni multiple comparisons test to compare the means between groups. The statistical analysis was performed using the statistical software PASW, version 18 for Windows (SPSS CPSC., Chicago, USA). Probability levels of 0.05 or less were considered significant.

\section{Results}

The study was conducted with 45 subjects, which included 30 type 2 diabetic patients and 15 healthy controls (Table 1). Amongst the 30 diabetic patients, 15 were non-hypertensive and the other 15 hypertensive. The study population consisted of $62 \%$ women versus $38 \%$ men. The average age of the diabetics was $59 \pm 9$ years ranging from 39 and 74 years. The mean age was $61 \pm 8$ years for hypertensive diabetics and $56 \pm 9$ years for non-hypertensive diabetics. For the controls, the average age was $33 \pm 7$ years ranging from 22 and 40 years. Mean body mass index (BMI) was 26.4 \pm 2.9 in diabetics versus $25.7 \pm 4.4$ in controls. The mean BMI was higher in women than men in both diabetics and controls.

Table 1. Demographic characteristics of the population.

\begin{tabular}{llllll}
\hline \multirow{2}{*}{ Parameters } & & Diabetics $(\mathbf{n}=\mathbf{3 0})$ & \multirow{2}{*}{ Diabetics $(\mathbf{n}=\mathbf{3 0})$} & \multirow{2}{*}{ Control $(\mathbf{n}=\mathbf{1 5})$} \\
\cline { 3 - 5 } & & Non-hypertensive $(\mathbf{n}=\mathbf{1 5})$ & Hypertensive $(\mathbf{n}=\mathbf{1 5})$ & & $24(80.0)$ \\
Sex n $(\%)$ & Female & $12(80.0)$ & $12(80.0)$ & $6(20.0)$ & $4(27.0)$ \\
& Male & $3(20.0)$ & $3(20.0)$ & $58 \pm 9$ & $25 \pm 3$ \\
\multirow{2}{*}{ Age (years) } & Female & $56 \pm 9$ & $60 \pm 9$ & $60 \pm 8$ & $35 \pm 7$ \\
& Male & $55 \pm 9$ & $65 \pm 6$ & $59 \pm 9$ & $33 \pm 7$ \\
& Total & $56 \pm 9$ & $61 \pm 8$ & $26.5 \pm 3.2$ & $28.3 \pm 4.5$ \\
BMI $\left(\mathrm{kg} / \mathrm{m}^{2}\right)$ & Female & $26.0 \pm 4.1$ & $27.1 \pm 1.9$ & $25.8 \pm 1.5$ & $24.8 \pm 4.2$ \\
& Male & $25.8 \pm 2.2$ & $25.9 \pm 1.0$ & $26.4 \pm 2.9$ & $25.7 \pm 4.4$ \\
\hline
\end{tabular}

According to the lipids profile in Table 2, only the HDL3C level was significantly higher in non-hypertensive diabetics compared to hypertensive diabetics $(\mathrm{p}<0.0001)$.

Table 2. Lipids values of diabetes group compared to control group.

\begin{tabular}{|c|c|c|c|c|c|c|}
\hline \multirow{2}{*}{$\begin{array}{l}\text { Parameters } \\
(\mathrm{M} \pm \mathrm{SD} \mathrm{mmol} / \mathrm{L})\end{array}$} & \multicolumn{3}{|l|}{ Diabetics $(n=30)$} & \multirow{2}{*}{ Diabetics $(n=30)$} & \multirow{2}{*}{ Control (n=15) } & \multirow{2}{*}{$\mathbf{p}$} \\
\hline & Non-Hypertensive $(n=15)$ & Hypertensive $(n=15)$ & $\mathbf{p}$ & & & \\
\hline Triglyceride & $1.22 \pm 0.59$ & $1.04 \pm 0.48$ & 0.36 & $1.13 \pm 0.54$ & $1.24 \pm 1.14$ & 0.55 \\
\hline Total Cholesterol & $5.56 \pm 1.22$ & $4.74 \pm 1.13$ & 0.06 & $5.15 \pm 1.23$ & $4.94 \pm 1.33$ & 0.60 \\
\hline LDL cholesterol & $3.80 \pm 1.34$ & $3.30 \pm 1.03$ & 0.26 & $3.55 \pm 1.20$ & $3.30 \pm 0.82$ & 0.47 \\
\hline HDL cholesterol & $1.20 \pm 0.35$ & $0.97 \pm 0.34$ & 0.07 & $1.09 \pm 0.36$ & $1.03 \pm 0.25$ & 0.56 \\
\hline HDL2 cholesterol & $0.26 \pm 0.23$ & $0.29 \pm 0.28$ & 0.75 & $0.28 \pm 0.25$ & $0.27 \pm 0.15$ & 0.68 \\
\hline
\end{tabular}

The types of dyslipidemia in diabetics were TC increase in level was higher in $86,67 \%$ of non-hypertensive diabetics $66.67 \%$, LDLC increase in $83.33 \%$ and HDLC decrease in $60 \%$ compare to hypertensive diabetics ( $p=0.02$ ) and the HDL3C (Table 3). The measurement of cholesterol sub-fractions showed a decrease of HDL2C in diabetics (76.67\%) whilst HDL3C level was increased in $73.33 \%$ of diabetics. The TC level increased in $100 \%$ of non-hypertensive diabetics versus $46.67 \%$ in hypertensive diabetics $(\mathrm{p}=0.003)$.

Table 3. Prevalence of dyslipidemia in the study population.

\begin{tabular}{|c|c|c|c|c|}
\hline \multirow{2}{*}{ Parameters } & \multicolumn{2}{|l|}{ Diabetics $(n=30)$} & \multirow{2}{*}{$\mathbf{p}$} & \multirow{2}{*}{$\begin{array}{l}\text { Diabetics }(n=30) \\
n(\%)\end{array}$} \\
\hline & Non-hypertensive Diabetics $(n=15)$ n $(\%)$ & Hypertensive Diabetics (n=15) n (\%) & & \\
\hline $\mathrm{TC}>4.67 \mathrm{mmol} / \mathrm{L}$ & $13(86.67)$ & $7(47.67)$ & 0.02 & $20(66.67)$ \\
\hline $\mathrm{LDL}>2.55 \mathrm{mmol} / \mathrm{L}$ & $14(93.33)$ & $11(73.33)$ & 0.32 & $25(83.33)$ \\
\hline $\mathrm{HDL}<1.15 \mathrm{mmol} / \mathrm{L}$ & $8(53.33)$ & $10(66.67)$ & 0.45 & $18(60.00)$ \\
\hline HDL2 $<0.43 \mathrm{mmol} / \mathrm{L}$ & $12(80.00)$ & $11(73.33)$ & 1 & $23(76.67)$ \\
\hline HDL3>0.68 mmol/L & $15(100.00)$ & $7(46.67)$ & 0.003 & $22(73.33)$ \\
\hline
\end{tabular}

In Table 4 , the prevalence of miRNA-33a (86.7\%) was significantly higher than miRNA-33b (53.3\%) in diabetics ( $p$ $=0.004)$. In the control group, the prevalence of miRNA-33a $(73.3 \%)$ was higher compared to $53.3 \%$ of miRNA-33b, without any significant difference $(p=0.25)$. Also there was no significant difference in the prevalences of miRNA-33a and miRNA-33b in non-hypertensive diabetics and in hypertensive diabetics $(\mathrm{p}=0.11)$ for both. 
Table 4. Prevalence of miRNA-33 in the study population.

\begin{tabular}{llllll}
\hline \multirow{2}{*}{ Parameters } & \multicolumn{2}{l}{ Diabetics $(\mathbf{n}=\mathbf{3 0})$} & \multirow{2}{*}{ Diabetics $(\mathbf{n}=\mathbf{3 0})$} & \multirow{2}{*}{ Control $(\mathbf{n}=\mathbf{1 5})$} \\
\cline { 2 - 5 } & Non-hypertensive $(\mathbf{n}=\mathbf{1 5})$ & Hypertensive $(\mathbf{n}=\mathbf{1 5})$ & $26(86.7)$ & $11(73.3)$ & $\mathbf{p}$ \\
\hline miRNA-33a & $13(86.7)$ & $13(86.7)$ & $16(53.3)$ & $8(53.3)$ & 0.49 \\
miRNA-33b & $8(53.3)$ & $8(53.3)$ & 0.004 & 0.25 & 1 \\
$\mathrm{p}$ & 0.11 & 0.11 & 11 & \\
\hline
\end{tabular}

The prevalence of dyslipidemia was significantly higher ( $\mathrm{p}$ $<0.0001$ ) in miRNA-33a positive subjects than miRNA-33a negative (Table 5). There was however no significant difference in the prevalence of dyslipidemia between miRNA-33b positive and miRNA-33b negative subjects. The comparison between miRNA-33a positive and miRNA-33b positive subjects showed a significant increase of dyslipidemia in miRNA-33a positive subjects. The dyslipidemic types in miRNA-33a positive diabetics were $90 \%$ hypercholesterolemia, $88 \%$ LDLC increase and $83.33 \%$ HDLC decrease. The measurement of the HDLC subclasses showed $82.6 \%$ HDL2C decrease and 90.91\% HDL3C increase. The HDL3C level increased in $100 \%$ of nonhypertensive diabetics versus $46.67 \%$ in hypertensive diabetics $(\mathrm{p}=0.003)$. The increase of HDL3C was $90.9 \%$ in miRNA33a positive versus $54.5 \%$ in miRNA33b positive ( $p$ $<0.006)$.

Table 5. Prevalence of dyslipidemia in patients with miRNA-33a/33b.

\begin{tabular}{|c|c|c|c|c|c|c|c|c|c|}
\hline Parameters (mmol/L) & $\begin{array}{l}\text { miR-33a+ } \\
\text { n (\%) }\end{array}$ & $\begin{array}{l}\text { miR-33a- } \\
\text { n (\%) }\end{array}$ & $\mathbf{P}$ & $\begin{array}{l}\text { miR-33b+ } \\
\text { n (\%) }\end{array}$ & $\begin{array}{l}\text { miR-33b- } \\
\text { n (\%) }\end{array}$ & $\mathbf{P}$ & $\begin{array}{l}\text { miR-33a+ } \\
\text { n (\%) }\end{array}$ & $\begin{array}{l}\text { miR-33b+ } \\
\text { n (\%) }\end{array}$ & $\mathbf{p}$ \\
\hline $\mathrm{TC}>4,67(\mathrm{n}=20)$ & $18(90.00)$ & $2(10.00)$ & $<0.0001$ & $10(50.00)$ & $10(50.00)$ & 1 & $18(90.00)$ & $10(50.00)$ & 0.005 \\
\hline $\mathrm{LDL}>2,55(\mathrm{n}=25)$ & $22(88.00)$ & $3(12.00)$ & $<0.0001$ & $12(48.00)$ & $13(52.00)$ & 0.77 & $22(88.00)$ & $12(48.00)$ & 0.002 \\
\hline HDL $<1,15(n=18)$ & $15(83.33)$ & $3(16.67)$ & $<0.0001$ & $11(61.11)$ & $7(38.89)$ & 0.18 & $15(83.33)$ & $11(61.11)$ & 0.13 \\
\hline HDL2 $<0,43(n=23)$ & $19(82.61)$ & $4(17.39)$ & $<0.0001$ & $14(60.87)$ & $9(39.13)$ & 0.14 & $19(82.61)$ & $14(60.87)$ & 0.1 \\
\hline HDL3>0,68 $(n=22)$ & $20(90.91)$ & $2(9.09)$ & $<0.0001$ & $12(54.55)$ & $10(45.45)$ & 0.54 & $20(90.91)$ & $12(54.55)$ & 0.006 \\
\hline
\end{tabular}

\section{Discussion}

According to the lipids profile in Table 2, only the HDL3C level was significantly higher in non-hypertensive diabetics compare to hypertensive diabetics ( $\mathrm{p}<0.0001$ ). This confirms the interest of measuring HDLC subclasses in the early detection of cardiovascular risks [6-7]. The prevalence of dyslipidemia in diabetics (Table 3) indicate TC increase in $66.67 \%$, LDLC increase in $83.33 \%$ and HDLC decrease in $60 \%$. The decrease of HDL2C was observed in $76.67 \%$ of diabetics and the HDL3C increase in $73.33 \%$. The TC level was higher in $86,67 \%$ of nonhypertensive diabetics compare to hypertensive diabetics $(p=0,02)$ and the HDLC3C level increased in $100 \%$ of nonhypertensive diabetics versus $46.67 \%$ in hypertensive diabetics $(\mathrm{p}=0.003)$.

The high prevalence of microRNA-33a (86.7\%) in diabetics compared to controls confirms the higher cardiovascular risk. Indeed, several studies have reported a high circulating rate of these microRNAs during acute myocardial infactus [12], acute coronary syndrome [13], stable coronary artery disease [14], heart failure [15], essential arterial hypertension [16] and stroke [17].

Among non-hypertensive diabetics and hypertensive diabetics, the prevalence of circulating microRNA-33a $(86.7 \%)$ was significantly higher $(p<0.004)$ than that of microRNA-33b (53.3\%). Among the controls the prevalence of microRNA-33a was $73.3 \%$ versus $53.3 \%$ for microRNA-33b but this difference is not statistically significant. The higher prevalence of microRNAs-33a in our series compared to microRNAs-33b is consistent with the literature. Indeed, the microRNA-33a which is involved in the repression of several cholesterol regulation genes [1] has been well conserved during evolution and is found in the vertebrate insects genome [18]. On the other hand, its isoform, microRNA-33b is only found in a limited number of species. Present in primates, microRNA-33b is not found in rodents which explains why it is little studied since the mouse is the animal model commonly used for the study of microRNAs.

The prevalence of dyslipidemia in microRNA-33a positive subjects was $90 \%$ for TC increase and $88 \%$ for LDLC increase. These prevalences were significantly higher $(p$ $<0.0001)$ compared to the negative microRNA-33a subjects. This confirms the significant role of microRNAs on lipid homeostasis [19-21].

The prevalence of dyslipidemia in our series is higher in microRNA-33a positive subjects than in microRNA-33b positive subjects with respectively $\mathrm{p}<0.005$ for TC increase, $\mathrm{p}$ $<0.002$ for LDLC increase and $p<0.006$ for the increase in HDL3C. The impact of microRNA-33a on dyslipidemia appears to be higher than microRNA-33b. This seems to be corroborated by the fact that no significant difference was observed between the frequency of dyslipidemia in microRNA-33b positive and microRNA-33b negative subjects.

The study reported that $83 \%$ of the microRNA-33a positive subjects had a low HDL level. The role of microRNAs in the decrease of HDLC has been reported by several authors who found that in vivo overexpression of microRNA-33 could lead to a $25 \%$ decrease in HDLC, whereas their inhibition increased the rate of circulating HDLC between 25 to $30 \%$ [2-4]. The mechanism of the regulation of microRNAs-33a/b on HDLC has been elucidated in vivo by studies which show that they are localized in the sterol-regulatory element-binding proteins 
(SREBPs) intron thus controlling the homeostasis of cholesterol [22]. In the human species, the microRNA-33a and microRNA-33b are encoded in the introns of SREBF2 and SREBF1 respectively [23-24], while in rodents there is a deletion of part of microRNA-33b which means that it cannot express itself. In addition, microRNAs-33a and 33b share the same basic sequence and differ only by 2 nucleotides. SREBF1 codes for the SREBP-1a and 1c proteins which mainly regulate lipogenic genes such as those of fatty acid synthase (AG synthase), Sterol-CoA desaturase (SCD) and acetylCoA carboxylase 1 (ACC1). The SREBF2 codes for the SREBP2 protein which mainly regulates the genes of HMGCR (Hydroxy methyl Gluraryl CoA Reductase) and the LDL receptor (LDLR) [25-27]. Several authors have shown that the target of microRNA-33a in vivo is the ATP-binding transporter 1 (ABCA1) [2-4, 22]. This molecule catalyzes the fixation of excess tissue cholesterol on apoAI to form nascent HDL and thus plays an important role in the efflux of cholesterol to the liver where it is degraded to bile acids, hence its anti-atherogenic role. It has also been reported that the treatment of mice with anti-microRNA-33a has resulted in an increase of HDL between 35 to $50 \%$ without affecting the other cholesterol lipoproteins [2-4]. Similarly, in microRNA33a -/- mice (knockout mice), a significant increase was observed in the expression of ABCA1 in the liver and macrophages as well as serum HDLC level of $25-40 \%$ [1, 22]. The increase in HDLC observed after treatment with antimicroRNA-33 is a promising issue for treatment or prevention in patients with low HDLC as their primary cardiovascular risk [18]. In contrast, the liver analysis and the HDLC measurement in microRNA-33b knock-in (KI) mice reported a $35 \%$ reduction in HDL cholesterol [27-29]. This makes the beneficial role of microRNA-33b in the regulation of HDLC disputable.

The measurement of HDLC subclasses showed that the decrease of HDL was due to the decrease of HDL2C with a prevalence of $83 \%$ in the microRNA-33a positive subjects. In contrast $90 \%$ of these microRNA-33a positive subjects had an increase in HDL3 cholesterol. This shows that the role of microRNA-33a on the decrease of HDLC seems to be mainly on the HDL2C fraction which is reported as the most protective fraction against cardiovascular diseases.

\section{Conclusion}

A high level of LDLC is a major cardiovascular risk. Treatment with statins that are inhibitors of hydroxy methyl glutaryl CoA reductase (HMGCR) drastically reduces this risk in current medical practice. However, there is still a risk in subjects with a low HDLC level (case of Tangier's disease). It is therefore necessary to explore other therapeutic routes. Thus, the study of the role of microRNAs in the regulation of cholesterol metabolism is a promising issue. The study of the impact of microRNAs on lipid profile reported that $83 \%$ of microRNA-33a positive subjects had low HDLC levels. This confirms the relationship between the presence of microRNA$33 \mathrm{a}$ and increased cardiovascular risk. The fractionation of
HDLC showed that the decrease particularly concerned the HDL2C and paradoxically $90 \%$ of the microRNA-33a positive subjects had an increase of HDL3C level. This observation supports the hypothesis that the role of microRNA-33a on HDLC level is mainly due to HDL2C. This observation suggests that research on drugs able to increase the HDLC levels based on microRNA regulation deserves to be refined to target the stimulation of HDL2C synthesis.

\section{Acknowledgements}

We thank the services of University Hospital, Yalgado Ouedraogo and the Regional Center of Blood Transfusion that helped us for the recruitment of patients and controls including the laboratory of biochemistry of the University Hospital, Yalgado Ouedraogo and the Centre of Biomolecular Research Pietro Annigoni (CEBRA).

\section{Conflict of Interest}

The authors declare that they have no conflict of interest.

\section{References}

[1] Rotllan N, Fernández-Hernando C. MicroRNA Regulation of Cholesterol Metabolism. Cholesterol. 2012: 847849. doi: 10.1155/2012/847849.

[2] Reddy LL, Shah SAV, Ponde CK, Rajani RM, Ashavaid TF. Circulating miRNA-33: a potential biomarker in patients with coronary artery disease. Biomarkers. 2019 Feb; 24 (1): 36-42. doi: 10.1080/1354750X.2018.1501760.

[3] Näär AM. miR-33: A Metabolic Conundrum. Trends Endocrinol Metab. 2018 Oct; 29 (10): 667-668. doi: 10.1016/j.tem.2018.04.004.

[4] Shao F, Wang X, Yu J, Shen K, Qi C, Gu Z. Expression of miR-33 from an SREBP2 intron inhibits the expression of the fatty acid oxidation-regulatory genes CROT and HADHB in chicken liver. Br Poult Sci. 2019 Apr; 60 (2): 115-124. doi: 10.1080/00071668.2018.1564242.

[5] Sun Y, Zhang D, Liu X, Li X, Liu F, Yu Y, Jia S, Zhou Y, Zhao Y.] Endoplasmic Reticulum Stress Affects Lipid Metabolism in Atherosclerosis Via CHOP Activation and Over-Expression of miR-33. Cell Physiol Biochem. 2018; 48 (5): 1995-2010. doi: 10.1159/000492522.

[6] Alice T. C. R. Kiba Koumaré, Absetou Guira, André Samandoulougou, Elie Kabré, Issaka Sondé, Talkmore Maruta, Jacques Simporé and Jean Sakandé. Plasma lipid profile including the high density lipoprotein (HDL) subclasses in hypertensive patients in Ouagadougou, Burkina Faso. Afr. J. Biochem. Res. 2015, 9 (3): 47-54, DOI: 10.5897/AJBR2015.0825.

[7] Alice TCR Kiba Koumaré, Assana Bouda, Hervé Tiénon, Elie Kabré, Issaka Sondé, Talkmore Maruta, Jacques Simporé and Jean Sakandé. Measurement of Cholesterol Sub-Fractions, High Density Lipoprotein 2 and High Density Lipoprotein 3 in Type 2 Diabetes Mellitus Patients in Burkina Faso (West Africa). Am. J. Biochem. Mol. Biol., 2015, ISSN 2150-4210 DOI: $10.3923 /$ ajbmb.2015. 
[8] Jomard A, Osto E Metabolism and Function of High-Density Lipoproteins (HDL). Praxis. 2019; 108 (7): 477-486. doi: 10.1024/1661-8157/a003241.

[9] Hirano, T., K. Nohtomi, S. Koba, A. Muroi and Y. Ito, 2008. A simple and precise method for measuring HDL-cholesterol subfractions by a single precipitation followed by homogenous HDL-cholesterol assay. J. Lipid Res., 49: 11301136.

[10] Srisawasdi P, Chaloeysup S, Teerajetgul Y, Pocathikorn A, Sukasem C, Vanavanan S, Kroll MH (2011). Estimation of plasma small dense LDL cholesterol from classic lipid measures. Am. J. Clin. Pathol. 136 (1): 20-9. http://dx.doi.org/10.1309/AJCPLHJBGG9L3ILS

[11] Koumaré Kiba ATCR, Sakandé LPL, Kabré E, Sondé I, Simporé J, Sakandé J (2015) Reference Ranges of Cholesterol Sub-Fractions in Random Healthy Adults in Ouagadougou, Burkina Faso. PLoS ONE 10 (1): e0116420. doi: 10.1371/journal. pone. 0116420 .

[12] Kuwabara Y, Ono K, Horie T, Nishi H, Nagao K, Kinoshita M, et al. Increased microRNA-1 and microRNA-133a levels in serum of pa- tients with cardiovascular disease indicate myocardial damage. Circ Cardiovasc Genet 2011; 4: 446-454.

[13] Widera C, Gupta SK, Lorenzen JM, Bang C, Bauersachs J, Bethmann K, et al. Diagnostic and prognostic impact of six circulating microRNAs in acute coronary syndrome. J Mol Cell Cardiol 2011; 51: 872-875.

[14] Fichtlscherer S, De Rosa S, Fox H, Schwietz T, Fischer A, Liebetrau $\mathrm{C}$, et al. Circulating microRNAs in patients with coronary artery disease. Circ Res 2010; 107: 677-684.

[15] Tijsen AJ, Creemers EE, Moerland PD, de Windt LJ, van der Wal AC, Kok WE, et al. Mir423-5p as a circulating biomarker for heart failure. Circ Res 2010; 106: 1035-1039.

[16] Li S, Zhu J, Zhang W, Chen Y, Zhang K, Popescu LM, et al. Signa- ture microRNA expression profile of essential hypertension and its novel link to human cytomegalovirus infection. Circulation 2011; 124: 175-184.

[17] Tan KS, Armugam A, Sepramaniam S, Lim KY, Setyowati $\mathrm{KD}$, Wang $\mathrm{CW}$, et al. Expression profile of microRNAs in young stroke patients. PLoS One 2009; 4: e7689, doi: 10.1371/journal.pone.0007689.

[18] Ouimet M, Hennessy EJ, van Solingen C, Koelwyn GJ, Hussein MA, Ramkhelawon B, Rayner KJ, Temel RE, Perisic L, Hedin U, Maegdefessel L, Garabedian MJ, Holdt LM, Teupser D, Moore KJ. miRNA Targeting of Oxysterol-Binding
Protein-Like 6 Regulates Cholesterol Trafficking and Efflux. Arterioscler Thromb Vasc Biol. 2016 May; 36 (5): 942-951. doi: 10.1161/ATVBAHA.116.307282.

[19] Rottiers V, Naar AM. MicroRNAs in metabolism and metabolic disorders. Nat Rev Mol Cell Biol 2012; 13: 239-250.

[20] Jeon TI, Osborne TF. miRNA and cholesterol homeostasis. Biochim Biophys Acta. 2016 Dec; 1861 (12 Pt B): 2041-2046. doi: 10.1016/j.bbalip.2016.01.005.

[21] Norata GD, Sala F, Catapano AL, Fernandez-Hernando C. MicroRNAs and lipoproteins: A connection beyond atherosclerosis? Atheroscle- rosis 2013; 227: 209-215.

[22] Horie T, Ono K, Horiguchi M, Nishi H, Nakamura T, Nagao K, et al. MicroRNA-33 encoded by an intron of sterol regulatory element- binding protein 2 (Srebp2) regulates HDL in vivo. Proc Natl Acad Sci USA 2010; 107: 17321-17326.

[23] Gerin I, Clerbaux LA, Haumont O, Lanthier N, Das AK, Burant CF, et al. Expression of miR-33 from an SREBP2 intron inhibits choles- terol export and fatty acid oxidation. $\mathrm{J}$ Biol Chem 2010; 285: 33652-33661.

[24] Brown MS, Ye J, Goldstein JL. Medicine: HDL miR-ed down by SREBP introns. Science 2010; 328: 1495-1496.

[25] Rui L. Energy metabolism in the liver. Compr Physiol. 2014 Jan; 4 (1): 177-97. doi: 10.1002/cphy.c130024. PMID: 24692138.

[26] Kim JH, Lee JM, Kim JH, Kim KR. Fluvastatin activates sirtuin 6 to regulate sterol regulatory element-binding proteins and AMP-activated protein kinase in HepG2 cells. Biochem Biophys Res Commun. 2018 Sep 10; 503 (3): 1415-1421. doi: 10.1016/j.bbrc.2018.07.057. Epub 2018 Aug.

[27] Gai Y, Li Y, Xu Z, Chen J Pseudoprotodioscin inhibits SREBPs and microRNA 33a/b levels and reduces the gene expression regarding the synthesis of cholesterol and triglycerides. Fitoterapia. 2019 Nov; 139: 104393. doi: 10.1016/j.fitote.2019.104393.

[28] Horie T, Nishino T, Baba O, Kuwabara Y, Nakao T, Nishiga M, et al. MicroRNA-33b knock-in mice for an intron of sterol regulatory element-binding factor 1 (Srebfl) exhibit reduced HDL-C in vivo. Sci Rep 2014; 4: 5312, doi: 10.1038/srep05312.

[29] Ono K, Horie T, Nishino T, Baba O, Kuwabara Y, Yokode M, Kita T, Kimura T. MicroRNA-33a/b in lipid metabolism-novel "thrifty" models. Circ J. 2015; 79 (2): 278-84. Doi 10.1253/circj.CJ-14-1252. 